\title{
X-ray Microdiffraction Characterization of Deformation Heterogeneities in BCC Crystals
}

K.R. Magid ${ }^{1}$, E.T. Lilleodden ${ }^{2}$, N. Tamura ${ }^{3}$, J.N. Florando ${ }^{4}$, D.H. Lassila ${ }^{4}$, M.M. LeBlanc ${ }^{4}$, R.I. Barabash $^{5}$ and J.W. Morris, Jr. ${ }^{1}$

${ }^{1}$ Department of Materials Science and Engineering, University of California-Berkeley, Berkeley, CA 94720, USA

${ }^{2}$ Institut für Materialforschung II, Forschungszentrum Karlsruhe, D-76021 Karlsruhe, Germany.

${ }^{3}$ Advanced Light Source, Lawrence Berkeley Laboratory, Berkeley, CA 94720, USA

${ }^{4}$ Engineering Directorate, Lawrence Livermore National Laboratory, Livermore, CA 94550, USA

${ }^{5}$ Oak Ridge National Laboratory, Oak Ridge, TN, USA

\begin{abstract}
The deformation behavior of BCC metals is being investigated by $\mathrm{x}$-ray microdiffraction measurements $(\mu \mathrm{XRD})$ for the purpose of characterizing the dislocation structure that results from uniaxial compression experiments. The high brilliance synchrotron source at the Advanced Light Source (Lawrence Berkeley National Lab) and the micron resolution of the focusing optics allow for the mapping of Laue diffraction patterns across a sample. These measurements are then analyzed in order to map the distribution of residual stresses in the crystal. An important finding is the observation of Laue spot "streaking", which indicates localized rotations in the lattice. These may represent an accumulation of same-sign dislocations. Theoretical modeling of the diffraction response for various slip systems is presented, and compared to experimental data. Preliminary results include orientation maps from a highly strained Ta bicrystal and a less highly strained Mo single crystal. The orientation maps of the bicrystal indicate a cell-like structure of dense dislocation walls. This deformation structure is consistent with previous OIM studies of the same crystal. The results suggest that $\mu$ XRD may be a particularly useful tool for microscale studies of deformation patterns in a multi-scale investigation of the mechanisms of deformation that ranges from macroscopic deformation tests to high resolution TEM studies of dislocation structures.
\end{abstract}

\section{INTRODUCTION}

The deformation of body-centered cubic (BCC) crystals is an important subject of current research, both for its inherent scientific interest [1] and because it is the focus of an extensive, on-going effort in the computer simulation of dislocation plasticity [2]. The modeling effort, in particular, has created a need for complementary experimental research to identify wellcharacterized examples that can be used as reliable test cases.

The construction of well-characterized examples of bulk deformation poses a number of experimental challenges whose solution would seem to require a "multi-scale" approach to the problem. The engineering result of plasticity is macroscopic deformation, which is tested by deforming bulk samples in large machines. The detailed mechanisms of plastic deformation ordinarily involve dislocation motion on the micro- to nanoscale, and are best studied with transmission electron microscopy. Given the usual heterogeneity of plastic deformation, however, it is always a challenge to select volumes for study in TEM that are actual participants in the current deformation. If this is not done, the detailed dislocation structures revealed by TEM, however interesting, may be irrelevant to the problem.

A number of experimental techniques have been developed in recent years that can help to bridge the experimental gap between macro- and nanoscale plastic deformation and focus the microscopic studies on the mechanisms that are most important. These include "speckle strain- 
gage" and high-resolution slip trace analysis that reveal the heterogeneous patterns of plastic deformation and identify the volumes in which deformation is actually occurring at any given time. The regions identified by these techniques can be studied in greater detail by orientation imaging microscopy (OIM) or scanning x-ray microdiffraction ( $\mu$ XRD) to observe patterns of deformation on the scale of microns and select interesting regions for high resolution study in TEM.

The work that is reported here is taken from collaborative work between the University of California, Berkeley and the Lawrence Livermore National Laboratory that is intended to develop probative methods for multiscale characterization of plastic deformation. We specifically discuss recent progress in the use of scanning x-ray microdiffraction as a fine-scale characterization tool. $\mu$ XRD and OIM methods work at roughly the same length scale, and reveal local plastic deformation by imaging the local reorientations of the crystal. However, $\mu$ XRD can also provide the mechanistic constituents of deformation by determining the active slip systems in a given region and map the changes active in slip systems over regions of the sample. It also offers the advantage of penetration into the sample to give some indication of patterns in the bulk. On the other hand, high-resolution $\mu$ XRD suffers from the disadvantage that it requires highly specialized synchrotron x-ray equipment, and can only be done in one of the few facilities that offer that capability. The work reported here was done at the Advanced Light Source (ALS) at Lawrence Berkeley National Lab (LBNL).

\section{EXPERIMENTAL DETAILS}

The X-ray microdiffraction beamline (7.3.3) of the Advanced Light Source (ALS) in Berkeley was used to collect polychromatic Laue patterns from highly purified BCC metal crystals. The beamline focuses the Synchrotron white beam $(6-14 \mathrm{keV})$ from a bending magnet using a pair of elliptically bent Kirkpatrick-Baez mirrors to achieve a spot size of approximately $1 \mu m \times 1 \mu m$ at the sample surface. A schematic of the beamline can be seen in figure 1 , and further details of the apparatus and experimental setup can be found elsewhere [3].

Two specific samples have been studied to date. The first was a tantalum bicrystal consisting of two single crystals diffusion bonded together to create a $90^{\circ}$ twist boundary and then compressed to 30-40\% strain. This sample was created for a previous study on the effect of a standard compression test on the boundary, and had been characterized with OIM [4]. The second sample was a single crystal of high purity molybdenum that was cut using electrical discharged machining $(\mathrm{EDM})$ to $5 \mathrm{~mm} \times 5 \mathrm{~mm} \times 15 \mathrm{~mm}$, and oriented for single slip before being strained to a maximum of $2 \%$ in compression. The compression test was done on the "6-degreeof-freedom" testing machine at LLNL [2], which allows for all rotation, tilt, and translation motion of the sample during compression. In addition to examining the bulk crystals, we also examined $3 \mathrm{~mm}$ cylinders cut from the samples along specific slip planes. The core samples were cut via EDM from bulk single crystals with normals along both the observed and theoretically most active slip planes. These core samples from the bulk crystals will also be used for future TEM studies.

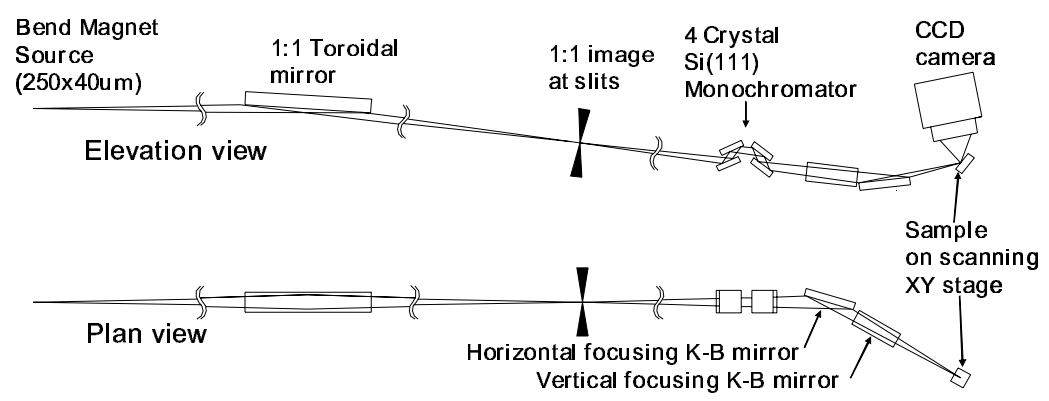

Figure 1: Schematic layout of beamline 7.3.3 at ALS. 
In the $\mu$ XRD experiments Laue patterns are automatically indexed and analyzed using software developed at ALS to determine the orientation and strain. The misorientation between any two grains as well as orientation variations within single grains can be determined with a precision of $0.01^{\circ}$. An unstrained crystal was used as a reference for the strain measurements of the deformed crystal, with a strain sensitivity of about $2 \times 10^{4}$. Additionally, the software can create simulated Laue patterns, which can be matched to the experimental data to determine the active slip systems. The simulation considers the consequence of edge dislocations as a lattice rotation, which appears as streaking in the Laue pattern in a direction characteristic of the active slip system. These simulations are performed with a method similar to approach described in [5, 6].

\section{RESULTS \& DISCUSSION}

\section{$\underline{\text { Tantalum Bicrystal }}$}

Prior work [4] had revealed unexpected behavior in the Ta bicrystal. In particular, the deformation pattern at the boundary differed significantly from that predicted with finite element techniques. We studied deformation patterns near the boundary to acquire additional information on this behavior.

The Laue patterns from this sample show extensive streaking characteristic of large plastic deformation and large dislocation density in the sample. The orientation maps of the bicrystal, as seen in figures 2 and 3, indicate the change in behavior in the bulk of a single crystal and across the boundary. Far from the boundary, a cell-like structure separated by dense dislocation walls is found. This deformation structure is consistent with previous OIM studies of similarly oriented and deformed single crystals [7]. The orientation map in figure 3 displays the cell structure far from the boundary, with misorientations between $5^{\circ}-11^{\circ}$ at the cell boundaries. Moreover, figure 3 shows how the average X-ray peak width can be used to detect sub-cell structures separated by high dislocation densities walls (up to $2(10)^{14} \mathrm{~m}^{-2}$ ). The cell boundaries correspond to localized high misorientation zones.

Close to the boundary, rotations on the order of $10^{\circ}$ are found both by EBSD and $\mu$ XRD. This perturbation to the cell structure presumably results from the constraint of the boundary resulting in geometrically necessary rotations required to keep the boundary intact. The saddleshaped boundary that is produced by the compression test differs from the simple bowed-out shape that crystal plasticity models predict. A systematic mapping of the deformation along the boundary is underway to clarify this behavior. Simulations of the shape of the Laue reflections are part of an on-going effort to map the active glide systems in the samples.
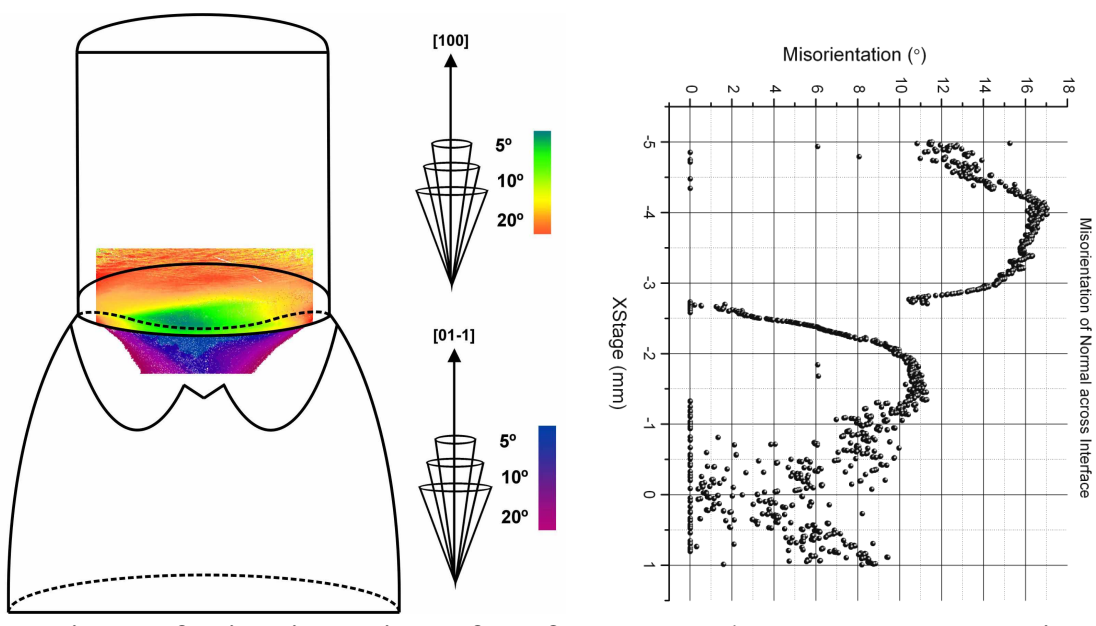

Figure 2: Comparison of misorientation of surface normal vector - representing lattice rotation across the interface in tantalum bicrystal from OIM (left) and $\mu$ XRD (right). 

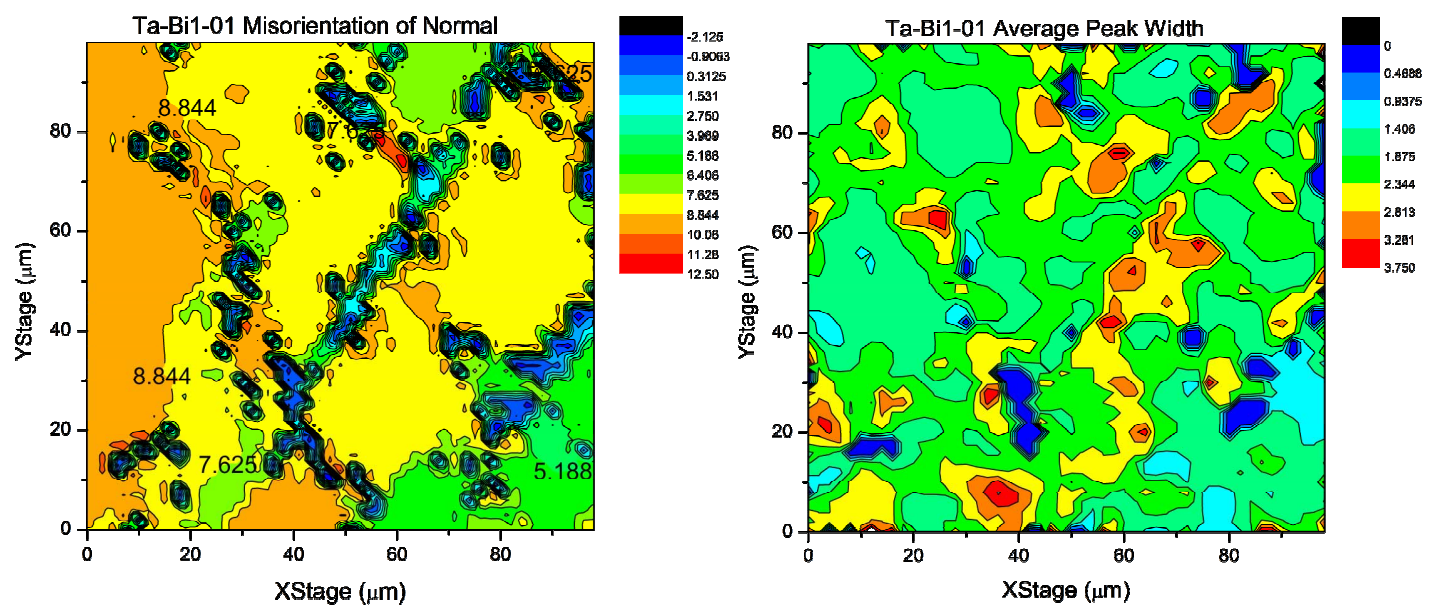

Figure 3: Misorientation of normal showing cell structure far from boundary (left) and average peak width as an indication of dislocation density and XRD resolved sub-cell structure in tantalum bicrystal (right).

\section{Molybdenum Single Crystals}

The Laue patterns in figures 4 and 5 show representative streaking from the bulk Mo crystal samples examined after compression testing and from core samples cut to reveal the slip planes in the interior. The streaking is heterogeneous in both types of samples. The streaking is much more extensive in the core samples cut from the interior than in the surface samples. The misorientation found in the core samples appears to be roughly periodic, as illustrated by the single reflection mosaic in figure 6 and the misorientation plot in figure 7 . It is not yet clear whether this periodicity is associated with slip-plane periodicity, wavy slip or the initial stages of cell formation. The issue is under further investigation

In the most streaked image from the bulk crystals, simulations have found the active slip systems to contain the burgers vectors and line directions of $\left[\begin{array}{ll}1 & 1\end{array}\right],\left[21 \frac{1}{1}\right]$ and $\left[\begin{array}{ll}1 & 1\end{array}\right],\left[\begin{array}{ll}2 & 1\end{array}\right]$. Laue image simulations concur with the slip system for the direction of the most extensive streaking.

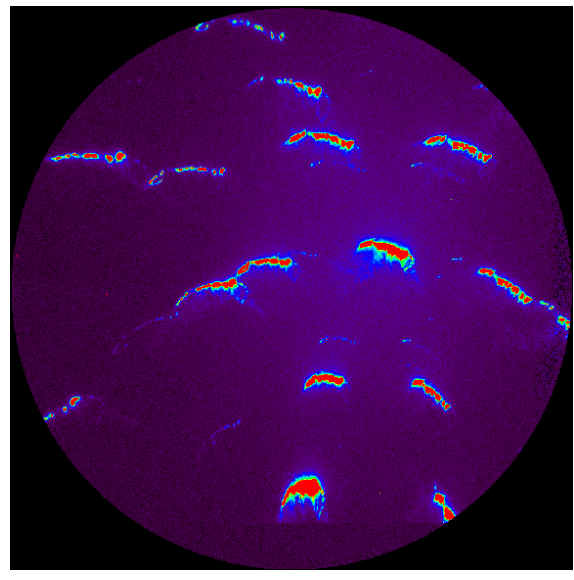

Figure 4: Laue pattern from core Mo sample showing extensive streaking.

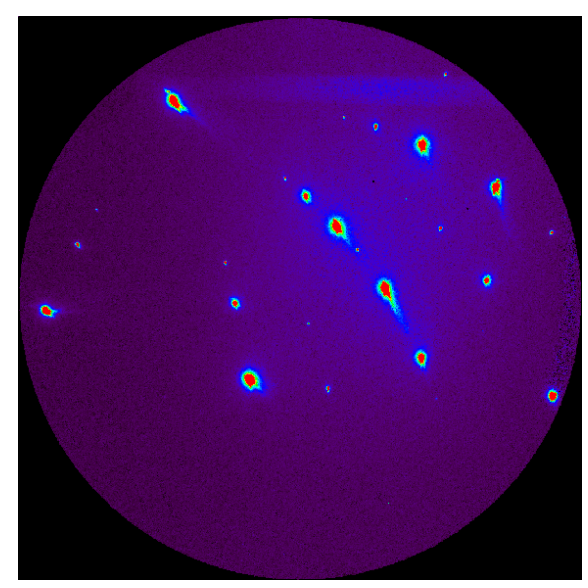

Figure 5: Laue pattern from bulk Mo sample showing more limited streaking. 


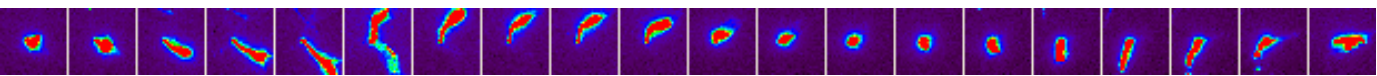

Figure 6: 024 spot mosaic from Laue patterns taken every $10 \mu \mathrm{m}$ showing large rotation in core samples machined from compressed bulk crystals.

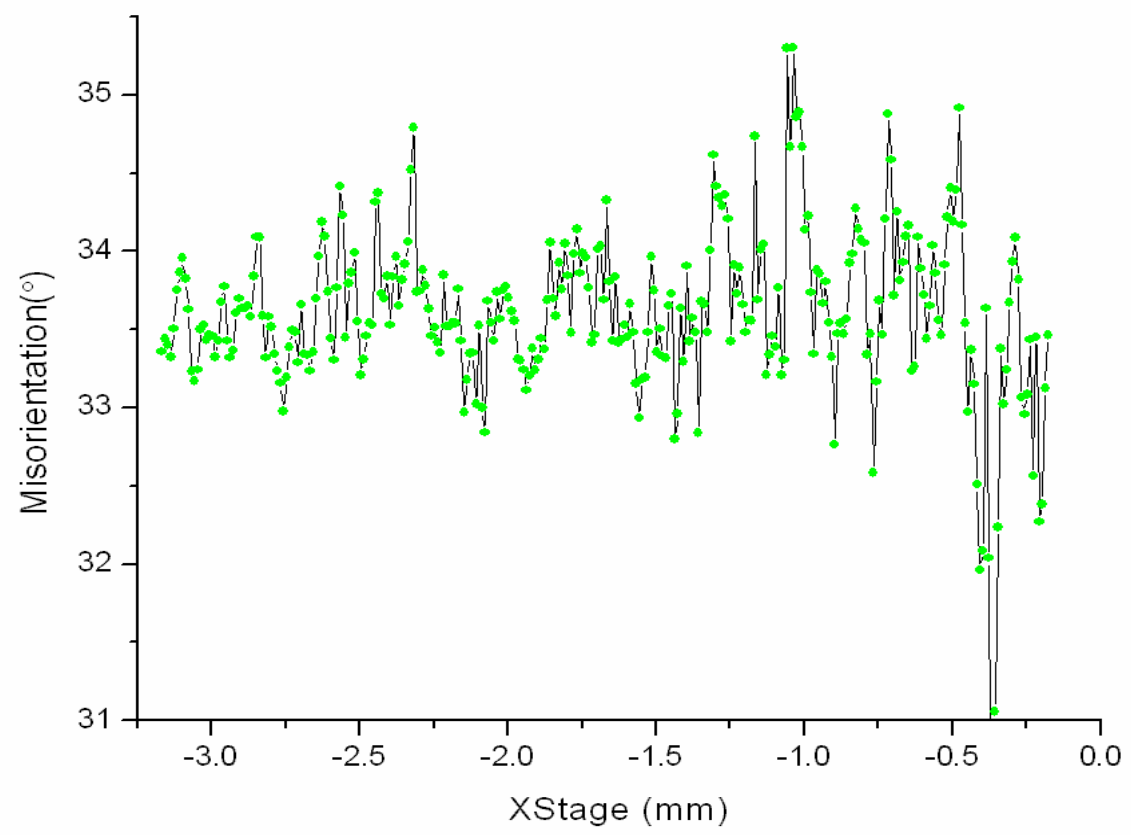

Figure 7: Misorientation of the sample normal taken from Laue patterns taken every $10 \mu \mathrm{m}$ showing roughly periodic lattice rotation corresponding to wavy slip.

\section{CONCLUSIONS}

$\mathrm{X}$-ray microdiffraction is being explored as a technique to characterize micro-patterns of plastic deformation in BCC crystals. Initial results on a Ta bicrystal and Mo single crystals deformed in compression shows that this technique can reveal interesting details of the dislocation structure, including misorientations and dominant slip systems. We believe that $\mu \mathrm{XRD}$ can be a useful characterization tool for analyzing deformation patterns on the scale intermediate between those of "speckle strain-gage" analysis and high resolution TEM. It complements OIM characterization methods in that it provides additional information, but requires experimental facilities (synchrotron x-ray facilities) that are much more difficult to access and use.

\section{ACKNOWLEDGEMENTS}

The Advanced light Source is supported by the Director, Office of Science, Office of Basic Energy Sciences, Materials Sciences Division, of the U.S. Department of Energy under Contract No. DE-AC03-76SF0098 at Lawrence Berkeley National Laboratory. This work was also performed under the auspices of the U.S. Department of Energy by University of California, Lawrence Livermore National Laboratory under Contract W-7405-Eng-48. We thank Geoff Campbell for access to the tantalum bicrystal and subsequent discussions as well as Alexander Ziegler for the previous OIM work. 


\section{REFERENCES}

1. J.W. Christian, Met. Trans. A, 14, 1237 (1983).

2. D.H. Lassila, M.M. Leblanc, and G. J. Kay, J. Eng. Mat. Tech., 124, 290 (2002).

3. N. Tamura, A. A. MacDowell, R. Spolenak, B. C. Valek, J. C. Bravman,W. L. Brown, R. S. Celestre, H. A Padmore, B.W. Batterman and J. R. Patel, J. Syn. Rad. 10, 137 (2003).

4. A. Zeigler, G.H. Campbell, M. Kumar, and J.S. Stolken in Multiscale Phenomena in Materials - Experiments and Modeling Related to Mechanical Behavior, edited by H.M. Zbib, D.H. Lassila, L.E. Levine, and K.J. Hemker, (Mater. Res. Soc. Symp. Proc. 779, San Francisco, CA, (2003) pp. 231-236.

5. R. Barabash, G. Ice, F. Walker, J. Appl. Physics, 93, 1457 (2003).

6. R. Barabash, G. Ice, B. Larson, G.M. Pharr, K.-S. Chung, W. Yang, Appl. Phys. Letters, 79, 749 (2001).

7. A.J. Schwartz., W.E. King, G.H. Campbell, J.S. Stolken, D.H. Lassila, S. Sun, B.L. Adams, ASME J. Eng. Mater. Tech., 121, 178 (1999). 